\title{
Translating the undruggable target
}

Clear cell renal cell carcinoma (ccRCC) is largely attributable to inactivating mutations in the von Hippel-Lindau tumour suppressor $\mathrm{pVHL}$, which drive accumulation and activation of the transcription factor hypoxia-inducible factor $2 \alpha$ (HIF2 $\alpha$ ). HIF2 $\alpha$, like many transcription factors, was considered undruggable until the discovery of a large cavity within the HIF2 $\alpha$ PAS-B domain capable of binding to small molecules. This breakthrough led to the identification of a series of selective HIF $2 \alpha$ antagonists. Three studies have now evaluated the anti-tumour activity of two of these compounds, PT2399 and the closely related analogue PT2385, in preclinical models of pVHL-mutant ccRCC.

What is the mechanism of HIF $2 \alpha$ antagonists? Using a $\mathrm{VHL}^{-/-} \mathrm{ccRCC}$ cell line, 786-O, both Cho et al. and Wallace et al. showed that treatment with PT2399 or PT2385 could disrupt the heterodimerization between HIF $2 \alpha$ and its transcriptional partner HIF1 $\beta$ (also known as ARNT), thereby preventing HIF2 $\alpha$ binding to DNA. Complementing these findings, Chen et al. demonstrated the same result with the treatment of patient-derived xenograft (PDX) ccRCC tumours. All three studies used quantitative PCR to show that both PT2399 and PT2385 suppressed the expression of a number of HIF2 $\alpha$-specific target genes, such as vascular endothelial growth factor A (VEGFA), cyclin D1 (CCND1) and erythropoietin (EPO), but importantly, not those of HIF1a, such as phosphoglycerate kinase 1 (PGK1).

What is the functional outcome of specific HIF $2 a$ transcriptional inhibition in vivo? Cho et al. and Wallace et al., using 786-O xenograft mouse models, saw that extended treatment with PT2399 or PT2385 caused tumour stasis and regression coinciding with decreased cell proliferation and decreased angiogenesis. PT2399 and PT2385 also substantially reduced tumour growth and decreased tumour vascular area in $V H L^{-/-}$ccRCC PDXs. Together these studies validate HIF2 $\alpha$ as a target driving pVHL-defective ccRCC.

Could HIF $2 \alpha$ antagonists be used as therapy for all $V H L^{-1-}$ ccRCCs? Surprisingly, Cho et al. and Chen et al. both observed that some VHL-mutant ccRCC cell lines and tumours were resistant to PT2399. These two groups came to the same conclusion that this resistance is probably the result of differential dependence on HIF2 $\alpha$, with both sensitive VHL-deficient ccRCC cell lines and PDX tumours having considerably higher HIF2 $\alpha$ levels. However, the responsiveness of $\mathrm{VHL}^{-/-} \mathrm{ccRCC}$ may not be exclusively ascribed to HIF2 $\alpha$ expression; Cho et al. identified two resistant ccRCC cell lines with high HIF2 $\alpha$ harbouring a p53 R248W mutation, suggesting that $\mathrm{p} 53$ status could also be a factor. In addition, Chen et al. found that even sensitive PDX tumours could gain resistance to PT2399 after prolonged exposure, with one tumour harbouring a PT2399 binding site mutation in HIF2 $\alpha$ and another a second site suppressor mutation in HIF1 $\beta$. This finding suggests that the design of complementary inhibitors may be required.

Do these HIF2 $\alpha$ antagonists represent an improvement over current approved first-line angiogenesis inhibitors for advanced ccRCC? Chen et al. and Wallace et al. compared the activity of PT2399 or PT2385 against sunitinib. Both HIF2 $\alpha$ antagonists were better tolerated, and showed greater efficacy

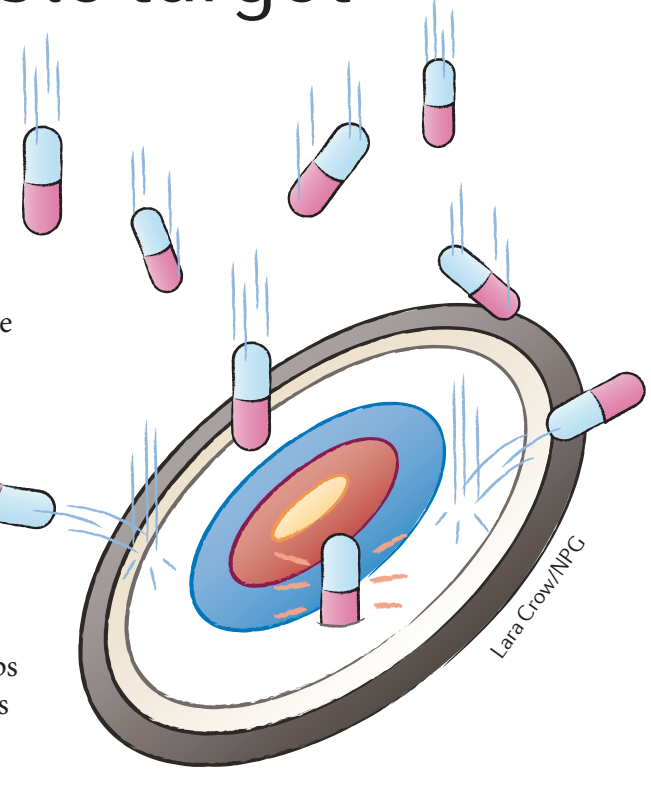

than sunitinib in reducing PDX tumour growth. Chen et al. even found that PT2399 inhibited tumour growth in some sunitinib-resistant PDX tumours.

The potential of PT2399 and PT2385 for clinical use is already evident, as Chen et al. demonstrated that a patient with extensively pretreated metastatic ccRCC, subsequently treated with PT2385 in a phase I trial, had a sensitive PDX tumour and exhibited progression-free survival for more than 11 months. However, these studies also underscore the need for predictive and pharmacodynamic biomarker-driven clinical trials, as patient responses are likely to depend, at least in part, on HIF $2 \alpha$ levels.

Anna Dart

ORIGINAL ARTICLES Chen, W. et al. Targeting renal cell carcinoma with a HIF-2 antagonist. Nature http://dx.doi.org/10.1038/nature19796 (2016) |Cho, H. et al. On-target efficacy of a HIF2a antagonist in preclinical kidney cancer models. Nature http://dx.doi.org/10.1038/nature19795 (2016) | Wallace, E. M. et al. A small-molecule antagonist of HIF2 $\alpha$ is efficacious in preclinical models of renal cell carcinoma. Cancer Res. 76, 5491-5500 (2016) 